\title{
Efetividade do protocolo para prevenção de lesões por pressão implantado em Unidade de Terapia Intensiva
}

Effectiveness of protocol implemented in an Intensive Care Unit for the prevention of pressure ulcers

Efectividad de protocolo para prevención de lesiones por presión implantada en Unidad de Terapia Intensiva

Odair Queiroz de Holandaํㅡ, Vanessa Amaral Oliveira²,

Flávia Emília Cavalcante Valença Fernandes ${ }^{3}$, Saulo Bezerra Xavier ${ }^{4}$, Rachel Mola $^{5}$

\footnotetext{
${ }^{1}$ Enfermeiro - Universidade de Pernambuco. Recife- PE

${ }^{2}$ Enfermeira - Universidade de Pernambuco. Recife- PE
}

${ }^{3}$ Enfermeira. Mestre em Gestão e Economia da saúde (UFPE). Docente Assistente do Curso de Bacharelado em Enfermagem - Universidade de Pernambuco. Recife- PE

${ }^{4}$ Enfermeiro. Mestre em Gestão e Economia da Saúde (UFPE). Universidade de Pernambuco. Recife- PE

${ }^{5}$ Enfermeira. Mestre em Hebiatria (FOP/UPE). Docente Assistente do Curso de Bacharelado em

Enfermagem - Universidade de Pernambuco. Recife- PE

\section{RESUMO}

Este estudo objetivou avaliar efetividade da implantação do protocolo para prevenção de lesão por pressão em unidade de terapia intensiva. Estudo quantitativo descritivo, analítico e documental. Foram aplicados testes Qui-quadrado de Pearson, Exato de Fisher para variáveis categóricas e Mann-Whitney para numéricas; significância de 5\% e intervalo de confiança de 95\%. A Amostra totalizou 566 pacientes, com médias de idade de 45 anos, escore da escala de Braden de 12,4 e tempo de internamento de 9,8 dias. Predominaram lesão por pressão sacral na admissão (11,1\%); do sexo masculino $(66,9 \%)$ e procedência da emergência $(54,0 \%)$. Houve redução significativa de lesão por pressão durante o internamento $(36,6 \%$ para 12,3\%), da média do tempo de internamento (11,4 para 7,1 dias), bem como o surgimento de lesão por pressão em regiões calcânea $(25,7 \%$ para $3,7 \%)$ e sacra $(9,9 \%$ para $2,8 \%)$. Foi comprovada a efetividade da implantação do protocolo para prevenção de lesão por pressão.

Palavras-chave: Lesão por Pressão. Enfermagem. Unidade de Terapia Intensiva. Internação hospitalar. 


\begin{abstract}
This study aimed to evaluate the effectiveness of protocol implantation for prevention of pressure injury in an intensive care unit. Descriptive, analytical and documentary quantitative study. Pearson's Chi-square test, Fisher's exact test for categorical variables and Mann-Whitney for numerical variables; significance of $5 \%$ and $95 \%$ confidence interval. The sample totaled 566 patients, with a mean age of 45 years, a Braden score of 12.4 and an inpatient time of 9.8 days. Prevalence of sacral pressure injury on admission (11.1\%); males (66.9\%) and emergence origin (54.0\%). There was a significant reduction in pressure injury during hospitalization (36.6\% to $12.3 \%$ ), mean length of hospital stay (11.4 to 7.1 days), as well as the appearance of pressure lesion in calcaneal regions $(25.7 \%$ to $3.7 \%)$ and sacra $(9.9 \%$ to $2.8 \%)$. The effectiveness of the implantation of the protocol for prevention of pressure injury has been proven.
\end{abstract}

Keywords: Pressure Ulcer. Nursing. Intensive Care Units. Hospitalization.

\title{
RESUMEN
}

Este estudio objetivó evaluar la efectividad de la implantación de protocolo para prevención de lesión por tensión en unidad de terapia intensiva. Estudio cuantitativo descriptivo, analítico y documental. Se aplicaron pruebas Qui-cuadrado de Pearson, Exacto de Fisher para variables categóricas y MannWhitney para numéricas; significante del 5\% y el intervalo de confianza del 95\%. La muestra tuvo un total de 566 pacientes, con promedios de edad de 45 años, puntuación de la escala de Braden de 12,4 y tiempo de internamiento de 9,8 días. Predominaron lesión por tensión sacral en la admisión $(11,1 \%)$; del sexo masculino $(66,9 \%)$ y procedencia de la emergencia $(54,0 \%)$. Se observó una reducción significativa de la lesión por tensión durante el internamiento (36,6\% para el 12,3\%), del promedio del tiempo de internamiento (11,4 a 7,1 días), así como el surgimiento de lesión por tensión en regiones calcáreas $(25,7 \%$ para el 3,7\%) y sacra (9,9\% para el 2,8\%). Se comprobó la efectividad de la implantación del protocolo para prevención de lesión por tensión.

Palabras clave: Úlcera por Presión. Enfermería. Unidades de Cuidados Intensivos. Hospitalización.

\section{INTRODUÇÃO}

A lesão por pressão (LPP) configura-se como um evento adverso no processo de atenção à saúde, definida como injúria que acomete a pele e os tecidos subjacentes, geralmente em uma área de proeminência óssea. A ocorrência de LPP está geralmente associada a um conjunto de fatores extrínsecos tais como: pressão, fricção, cisalhamento e umidade; e intrínsecos como: déficit nutricional, presença de edema, idade, imobilidade e patologia de base $^{1-4}$.

O desenvolvimento deste tipo de ferida pode refletir em inúmeras consequências negativas. Para o paciente e a família, resulta no sofrimento físico e emocional, além de elevar o risco de complicações e comorbidades a curto, médio e longo prazo. Já para o sistema de saúde, resulta em maiores custos frente à necessidade da permanência prolongada no serviço de saúde, bem como maior investimento de recursos materiais e humanos de assistência ao paciente ${ }^{4}$.

Os registros de ocorrência de LPP em pacientes críticos no Brasil variam entre $11 \%$ e $88 \%{ }^{1}$, incidência acima do publicado em literatura internacional, que 
é de $4,9 \%$ a $25,1 \%$. Segundo o relatório nacional de incidentes relacionados à assistência à saúde, entre janeiro de 2014 e julho de 2017, foram notificados $134.50^{1}$ incidentes; destes, $17,6 \%$ são referentes à ocorrência de LPP, sendo o estágio III prevalente nas notificações dos Núcleos de Segurança do Paciente (NSP) dos serviços de saúde do país ${ }^{6}$.

A partir do reconhecimento da magnitude desta problemática, foi instituído em 2013, pelo Ministério da Saúde, o Programa Nacional de Segurança do Paciente (PNSP), cujo objetivo principal é contribuir para o cuidado de qualidade em todos os estabelecimentos de saúde ${ }^{7}$.

Neste contexto, a equipe multidisciplinar tem papel fundamental na prevenção e manejo das LPP, independentemente do nível de atenção à saúde ${ }^{6}$. A equipe de enfermagem torna-se referência na prevenção e redução dos riscos de desenvolvimento de LPP, visto que prestam assistência direta e permanente ao paciente crítico $^{2,8}$. Além disso, o enfermeiro como líder da equipe de enfermagem atua como mediador na tomada de decisões, aprimora suas habilidades assistenciais e de gerenciamento do serviço, a fim de garantir os recursos para segurança do paciente e melhor qualidade do cuidado?.

A utilização de instrumentos que visem a prevenção, identificação, acompanhamento e controle de eventos adversos tais como a ocorrência de LPP, são ferramentas importantes no cuidado, uma vez que o não desenvolvimento representa um indicador de qualidade do serviço de saúde ${ }^{9,10}$. Os protocolos são orientadores da assistência, direcionando e normatizando uma conduta a ser tomada, diminuindo assim o tempo de internação, o índice de mortalidade, os custos terapêuticos, a carga de trabalho da equipe e em especial o sofrimento ao paciente e aos familiares ${ }^{9}$.

A Unidade de Terapia Intensiva (UTI) é o setor hospitalar voltado ao atendimento do paciente crítico que comumente está sujeito a desenvolver LPP frente a um conjunto de fatores de risco, representando um universo ideal para seu surgimento ${ }^{11}$. A implementação de medidas preventivas guiadas por protocolos, podem auxiliar na mudança e avaliação contínua das atitudes assistenciais antes não padronizadas ou priorizadas. Assim, o objetivo desta pesquisa foi avaliar a efetividade da implantação de protocolo para prevenção de LPP em UTI.

\section{MÉTODO}

Estudo quantitativo descritivo, analítico e documental realizado na UTI do Hospital Universitário, localizado no município de Petrolina-PE, após a implantação de um protocolo para prevenção de LPP. Os dados foram coletados nos livros de registros e o protocolo para prevenção de LPP, no período de outubro de 2015 a dezembro de 2016, totalizando 566 pacientes.

Foram consideradas as seguintes variáveis: 1) caracterização sociodemográfica e clínica: sexo; média de idade em anos; média do escore da Escala de Braden; lesão por pressão na admissão; surgimento de lesão por pressão durante o internamento; média do tempo de internamento em dias; estado de procedência; setor de procedência; hipótese diagnóstica; desfecho clínico e setor de destino. 2) caracterização das LPP segundo a região corporal no momento da admissão e durante o internamento: sacra; calcânea; occipital, trocantérica e outros locais. 3) medidas preventivas de LPP durante o internamento: aplicação de aliviadores de pressão na região calcânea; de placas de hidrocolóide regular na região sacra e aplicação preventiva de coxins.

A classificação do risco de desenvolver LPP teve como referência a escala de Braden, considerando a classificação de 06 a 11 - risco alto, 12 a 17 - risco moderado e 18 a 20 - risco baixo.

Os dados foram analisados por meio do software estatístico Stata versão 14.0 e o Microsoft office Excel ${ }^{\circledR}$ 2013. Foram aplicados os testes Qui-quadrado de Pearson, Exato de Fisher para as variáveis categóricas e Mann-Whitney para variáveis numéricas que não 
apresentaram distribuição normal pelo teste Shapiro Wilk, sendo considerado nível de significância de 5\% e intervalo de confiança (IC) de 95\%.

A pesquisa respeitou os termos estabelecidos pela Resolução 466/2012 do Conselho Nacional de Saúde, sendo aprovada pelo Comitê de Ética em Pesquisa da Universidade de Pernambuco, parecer № 2.265.019. Por se tratar de dados secundários, não foi necessário o uso do termo de consentimento livre e esclarecido.

\section{RESULTADOS}

Dos 566 pacientes analisados, a média de idade foi de
45,0 anos (Desvio Padrão-DP 19,6 anos; IC95\% 43,346,6), a média do escore da escala de Braden foi de 12,4 (DP 2,9; IC95\% 12,2-12,6) e a média do tempo de internamento foi de 9,8 dias (DP 10,2 dias; IC95\% 8,910,6).

Com relação às características sociodemográficas e clínicas, houve prevalência do sexo masculino (66,9\%), procedentes do setor da Emergência (54,0\%), com hipótese diagnóstica de natureza clínica $(38,4 \%)$ e sem presença de LPP no momento da admissão $(83,0 \%)$. Com relação ao desfecho clínico dos pacientes, a maioria teve alta da UTI para outro setor do referido hospital $(68,2 \%)$, predominando o setor de clínica cirúrgica $(38,5 \%)$ (Tabela 1$)$.

Tabela 1 - Caracterização sociodemográfica e clínica dos pacientes internados na unidade de terapia intensiva. Hospital Universitário, 2015 - 2016. Petrolina - PE.

\begin{tabular}{|c|c|c|}
\hline & n (\%) & IC 95\% \\
\hline \multicolumn{3}{|l|}{ Sexo $(n=562)$} \\
\hline Feminino & $186(33,1)$ & $29,3-37,1$ \\
\hline Masculino & $376(66,9)$ & $62,9-70,7$ \\
\hline \multicolumn{3}{|c|}{ Setor de procedência $(n=87)$} \\
\hline Emergência & $47(54,0)$ & $43,3-64,4$ \\
\hline Bloco Cirúrgico & $32(36,8)$ & $27,2-47,6$ \\
\hline Clínicas & $6(6,9)$ & $3,1-14,7$ \\
\hline Transferência externa & $2(2,3)$ & $0,6-9,0$ \\
\hline \multicolumn{3}{|c|}{ Hipótese diagnóstica ( $\mathrm{n}=547)$} \\
\hline Clínico & $210(38,4)$ & $34,4-42,6$ \\
\hline Causas externas & $167(30,5)$ & $26,8-34,5$ \\
\hline Cirúrgico & $170(31,1)$ & $27,3-35,1$ \\
\hline \multicolumn{3}{|c|}{ Lesão por pressão na admissão $(\mathrm{n}=566)$} \\
\hline Não & $470(83,0)$ & $79,7-85,9$ \\
\hline Sim & $96(17,0)$ & $14,1-20,3$ \\
\hline \multicolumn{3}{|l|}{ Desfecho clínico $(\mathrm{n}=444)$} \\
\hline Alta para outro setor & $303(68,2)$ & $63,7-72,4$ \\
\hline Alta por óbito & $141(31,8)$ & $27,6-36,3$ \\
\hline \multicolumn{3}{|l|}{ Setor de destino $(\mathrm{n}=444)$} \\
\hline Óbito & $141(31,8)$ & $27,6-36,3$ \\
\hline Clínica médica & $98(22,1)$ & $18,4-26,2$ \\
\hline Clínica cirúrgica & $171(38,5)$ & $34,1-43,1$ \\
\hline Clinica ortopédica & $12(2,7)$ & $1,5-4,7$ \\
\hline Bloco cirúrgico & $1(0,2)$ & $0,0-1,6$ \\
\hline Sala amarela & $7(1,6)$ & $0,8-3,3$ \\
\hline Transferência & $11(2,5)$ & $1,4-4,4$ \\
\hline Alta hospitalar & $3(0,7)$ & $0,2-2,1$ \\
\hline
\end{tabular}


Entre os pacientes que possuíam LPP no momento da admissão, a região corporal mais acometida foi a sacra (11,1\%). O surgimento de LPP durante o internamento foi presente em 22,3\% dos pacientes (IC95\% 19,0-25,9), sendo a região calcânea prevalente (12,4\%).

Após a implantação do protocolo no setor, houve a aplicação de aliviadores de pressão na região calcânea e de placas de hidrocoloide regular na região sacra (72,2\%; IC95\% 68,4-75,8). Não houve aplicação preventiva de coxins para a maioria dos pacientes (94,2\%; IC95\% 91,9-95,8) (Tabela 2).

A análise dos fatores associados à implantação do protocolo foi referente ao último trimestre dos anos de 2015 e 2016, revelando uma diferença significativa do surgimento das LPP durante o internamento do paciente, com uma redução de $36,6 \%$ para $12,3 \%(\mathrm{p}<0,001)$. Além dessas, outras variáveis que apresentaram significância estatística foram: a média do tempo de internamento, em que houve redução de 11,4 para 7,1 dias ( $\mathrm{p}<0,001)$, e a hipótese diagnóstica, que apresentou mudança na incidência, inicialmente, por causas externas $(44,4 \%)$ passando para cirúrgico $(50,0 \%)(\mathrm{p}<0,001)$ (Tabela 3$)$.

No que diz respeito à associação da caracterização das LPP, segundo a região corporal dos pacientes internados na UTI, no momento da admissão e durante o internamento, as variáveis que apresentaram diferença significativa foram a aplicação preventiva de coxins, havendo uma redução na utilização, passando de $21,8 \%$ para $0 \%$ ( $<<0,001)$ e o surgimento de LPP em calcâneos e sacra, durante o período de internamento, que declinaram de $25,7 \%$ para $3,7 \%(\mathrm{p}<0,001)$ e $9,9 \%$ para $2,8 \%(\mathrm{p}=0,036)$ respectivamente (Tabela 4$)$.

Tabela 2 - Caracterização das lesões por pressão segundo a região corporal dos pacientes internados na unidade de terapia intensiva no momento da admissão e durante o internamento. Hospital Universitário, out/2015 dez/2016. Petrolina - PE.

\begin{tabular}{|c|c|c|c|c|}
\hline \multirow[t]{2}{*}{ Região corporal } & \multicolumn{2}{|c|}{ Na admissão } & \multicolumn{2}{|c|}{ Durante o internamento } \\
\hline & n (\%) & IC 95\% & & IC 95\% \\
\hline \multicolumn{5}{|l|}{ Sacra } \\
\hline Não & $503(88,9)$ & $86,0-91,2$ & $518(91,7)$ & $89,1-93,7$ \\
\hline Sim & $63(11,1)$ & $8,8-14,0$ & $47(8,3)$ & $6,3-10,9$ \\
\hline \multicolumn{5}{|l|}{ Calcânea } \\
\hline Não & $531(93,8)$ & $91,5-95,5$ & $495(87,6)$ & $84,6-90,1$ \\
\hline Sim & $35(6,2)$ & $4,5-8,5$ & $70(12,4)$ & $9,9-15,4$ \\
\hline \multicolumn{5}{|l|}{ Occipital } \\
\hline Não & $563(99,5)$ & $98,4-99,8$ & $544(96,3)$ & $94,4-97,6$ \\
\hline Sim & $3(0,5)$ & $0,2-1,6$ & $21(3,7)$ & $2,4-5,6$ \\
\hline \multicolumn{5}{|l|}{ Trocantérica } \\
\hline Não & $566(100,0)$ & - & $563(99,7)$ & $98,6-99,9$ \\
\hline Sim & - & - & $2(0,4)$ & $0,1-1,4$ \\
\hline \multicolumn{5}{|l|}{ Outros locais* } \\
\hline Não & $548(96,8)$ & $95,0-98,0$ & $540(95,6)$ & $93,5-97,0$ \\
\hline $\operatorname{Sim}$ & $18(3,2)$ & $2,0-5,0$ & $25(4,4)$ & $3,0-6,5$ \\
\hline
\end{tabular}

*Maléolo, orelhas, lábios, nádegas, olecrano/ cotovelos. 
Tabela 3 - Associação das características sociodemográficas e clínicas dos pacientes internados na unidade de terapia intensiva. Hospital Universitário, 2015-2016. Petrolina - PE.

\begin{tabular}{|c|c|c|c|c|c|}
\hline \multirow{2}{*}{ Variáveis } & \multicolumn{2}{|c|}{2015} & \multicolumn{2}{|c|}{2016} & \multirow[b]{2}{*}{ p-valor } \\
\hline & Média & IC95\% & Média & IC95\% & \\
\hline Idade & 43,2 & $39,3-47,0$ & 42,9 & $39,1-46,8$ & $0,975^{\star \star *}$ \\
\hline Escala de Braden & 12,1 & $11,7-12,6$ & 12,7 & $12,1-13,2$ & $0,219^{\star \star \star}$ \\
\hline Tempo de internamento & 11,4 & $9,4-13,5$ & 7,1 & $5,8-8,4$ & $<0,001^{\star \star \star}$ \\
\hline \multirow[t]{2}{*}{ Variáveis } & 2015 & & 2016 & total & \multirow[b]{2}{*}{ p-valor } \\
\hline & n (\%) & & n (\%) & n (\%) & \\
\hline \multicolumn{6}{|l|}{ Sexo } \\
\hline Feminino & $27(27,6)$ & & $37(34,9)$ & $64(31,4)$ & \multirow{2}{*}{$0,258^{*}$} \\
\hline Masculino & $71(72,5)$ & & $69(65,1)$ & $140(68,6)$ & \\
\hline \multicolumn{6}{|l|}{ Hipótese diagnóstica } \\
\hline Clínico & $33(36,7)$ & & $29(27,4)$ & $62(31,6)$ & \multirow{3}{*}{$<0,001^{*}$} \\
\hline Causas externas & $40(44,4)$ & & $24(22,6)$ & $64(32,7)$ & \\
\hline Cirúrgico & $17(18,9)$ & & $53(50,0)$ & $70(35,7)$ & \\
\hline \multicolumn{6}{|c|}{ Lesão por pressão na admissão } \\
\hline Não & $77(76,2)$ & & $89(84,0)$ & $166(80,2)$ & \multirow{2}{*}{$0,163^{*}$} \\
\hline $\operatorname{Sim}$ & $24(23,8)$ & & $17(16,0)$ & $41(19,8)$ & \\
\hline \multicolumn{6}{|c|}{$\begin{array}{l}\text { Lesão por pressão durante o } \\
\text { internamento }\end{array}$} \\
\hline Não & $64(63,4)$ & & $93(87,7)$ & $157(75,9)$ & \multirow{2}{*}{$<0,001^{*}$} \\
\hline Sim & $37(36,6)$ & & $13(12,3)$ & $50(24,2)$ & \\
\hline \multicolumn{6}{|l|}{ Desfecho clínico } \\
\hline Alta para outro setor & $3(50,0)$ & & $78(76,5)$ & $81(75,0)$ & \multirow[t]{2}{*}{$0,164^{* *}$} \\
\hline Alta por óbito & $3(50,0)$ & & $24(23,5)$ & $27(25,0)$ & \\
\hline
\end{tabular}

${ }^{*}$ Qui quadrado; ${ }^{* \star}$ Exato de Fisher; ${ }^{* \star}$ Mann-Whitney. 
Tabela 4 - Associação da caracterização das lesões por pressão segundo a região corporal dos pacientes internados na unidade de terapia intensiva no momento da admissão e durante o internamento. Hospital Universitário, 2015-2016. Petrolina - PE.

\begin{tabular}{|c|c|c|c|c|}
\hline \multirow[t]{2}{*}{ Variáveis } & 2015 & 2016 & total & \multirow[b]{2}{*}{ p-valor } \\
\hline & n (\%) & n (\%) & n (\%) & \\
\hline \multicolumn{5}{|c|}{ Aplicação de medidas preventivas } \\
\hline Não & $14(13,9)$ & $25(23,6)$ & $39(18,8)$ & \multirow{2}{*}{$0,074^{*}$} \\
\hline Sim & $87(86,1)$ & $81(76,4)$ & $168(81,2)$ & \\
\hline \multicolumn{5}{|c|}{ Aplicação preventiva de coxins } \\
\hline Não & $79(78,2)$ & $106(100,0)$ & $185(89,4)$ & \multirow{2}{*}{$<0,001^{*}$} \\
\hline $\operatorname{Sim}$ & $22(21,8)$ & $0(0,0)$ & $22(10,6)$ & \\
\hline \multicolumn{5}{|c|}{ Região corporal na admissão: } \\
\hline \multicolumn{5}{|l|}{ Sacra } \\
\hline Não & $82(81,2)$ & $96(90,6)$ & $178(86,0)$ & \multirow{2}{*}{$0,052^{*}$} \\
\hline Sim & $19(18,8)$ & $10(9,4)$ & $29(14,0)$ & \\
\hline \multicolumn{5}{|l|}{ Calcânea } \\
\hline Não & $91(90,1)$ & $101(95,3)$ & $192(92,8)$ & \multirow{2}{*}{$0,150^{*}$} \\
\hline $\operatorname{Sim}$ & $10(9,9)$ & $5(4,7)$ & $15(7,3)$ & \\
\hline \multicolumn{5}{|l|}{ Occipital } \\
\hline Não & $100(99,0)$ & $105(99,1)$ & $205(99,0)$ & \multirow{2}{*}{$1,000^{* *}$} \\
\hline $\operatorname{Sim}$ & $1(1,0)$ & $1(0,9)$ & $2(1,0)$ & \\
\hline \multicolumn{5}{|l|}{ Trocantérica } \\
\hline Não & $101(100,0)$ & $106(100,0)$ & $207(100,0)$ & \multirow{2}{*}{-} \\
\hline Sim & $0(0,0)$ & $0(0,0)$ & $0(0,0)$ & \\
\hline \multicolumn{5}{|c|}{ Outros locais } \\
\hline Não & $99(98,0)$ & $100(94,3)$ & $199(96,1)$ & \multirow{2}{*}{$0,281^{\star *}$} \\
\hline Sim & $2(2,0)$ & $6(5,7)$ & $8(3,9)$ & \\
\hline \multicolumn{5}{|c|}{$\begin{array}{l}\text { Região corporal durante o } \\
\text { internamento: }\end{array}$} \\
\hline \multicolumn{5}{|l|}{ Sacra } \\
\hline Não & $91(90,1)$ & $103(97,2)$ & $194(93,7)$ & \multirow{2}{*}{$0,036^{*}$} \\
\hline Sim & $10(9,9)$ & $3(2,8)$ & $13(6,3)$ & \\
\hline \multicolumn{5}{|l|}{ Calcânea } \\
\hline Não & $75(74,2)$ & $102(96,2)$ & $177(85,5)$ & \multirow{2}{*}{$<0,001^{*}$} \\
\hline Sim & $26(25,7)$ & $4(3,7)$ & $30(14,4)$ & \\
\hline \multicolumn{5}{|l|}{ Occipital } \\
\hline Não & $97(96,0)$ & $102(96,2)$ & $199(96,1)$ & \multirow{2}{*}{$1,000^{\star *}$} \\
\hline Sim & $4(4,0)$ & $4(3,8)$ & $8(3,9)$ & \\
\hline \multicolumn{5}{|c|}{ Trocantérica } \\
\hline Não & $100(99,0)$ & $106(100,0)$ & $206(99,5)$ & \multirow{2}{*}{$0,488^{* *}$} \\
\hline Sim & $1(1,0)$ & $0(0,0)$ & $1(0,5)$ & \\
\hline
\end{tabular}

${ }^{*}$ Qui quadrado; ${ }^{* \star}$ Exato de Fisher. 


\section{DISCUSSÃO}

Esta pesquisa foi representada por indivíduos com média de idade 45,0 anos, do sexo masculino. Embora estudos nacionais e internacionais realizados sobre o levantamento do perfil de pacientes com LPP, em UTI, corroborem com a prevalência do sexo masculino ${ }^{12,13}$, a idade envolve geralmente pacientes idosos ${ }^{14,15}$. A diferença na média de idade desta pesquisa pode estar relacionada à caracterização da população atendida na instituição analisada, que é referência para causas externas como acidentes de transporte terrestre, envolvendo comumente indivíduos adultos jovens ${ }^{16,17}$.

A média do escore da escala de Braden configurou uma amostra dentro da faixa de risco moderado de desenvolver LPP ${ }^{18}$, sendo o uso de escalas de risco uma ferramenta fundamental na assistência ao paciente submetido a cuidados intensivos, visto que são mais susceptíveis a injúrias teciduais ${ }^{12}$. Com relação ao tempo de internamento, a média de 9,8 dias esteve na faixa encontrada na descrição de outras pesquisas na área ${ }^{13-14}$.

O setor de procedência, da maioria dos pacientes nesta pesquisa, foi a emergência, seguido pelo centro cirúrgico. A instituição hospitalar analisada admite um grande quantitativo de paciente com diagnóstico clínico $^{13,19}$, de politrauma e traumato-ortopedia ${ }^{17,19}$ e oferece cirurgia de grande porte, desta forma, os pacientes admitidos na UTI geralmente seguem este fluxo de atendimento ${ }^{19}$. A alta hospitalar para outros setores do hospital foi o desfecho clínico prevalente dos pacientes, seguido de alta por óbito. O desfecho clínico pode ter relação com o perfil da clientela atendida, podendo haver variações dos resultados entre outras pesquisas realizadas ${ }^{14-15}$.

No momento da admissão na UTI, a maioria dos pacientes desta pesquisa não apresentava LPP, e entre os portadores admitidos com alguma LPP a região sacra foi prevalente ${ }^{14}$, provavelmente em decorrência da característica do setor de origem, onde a maioria procedeu da emergência e do bloco cirúrgico, e os esforços são destinados prioritariamente à estabilização do quadro clínico do paciente ${ }^{17}$. Além disso, especificamente na instituição avaliada, o único setor que possui um protocolo voltado à prevenção de LPP é a UTI. Já durante o período de internamento na UTI, a região calcânea foi prevalente no surgimento de LPP, mesmo com a implementação do protocolo, o que inclui, entre as medidas, a aplicação de protetores de calcâneo. Entretanto, deve-se considerar a pluralidade de fatores intrínsecos e extrínsecos para o desenvolvimento de $\mathrm{LPP}^{14,21}$, principalmente em pacientes críticos.

Com relação à associação das características sociodemográficas e clínicas dos pacientes internados na UTI houve redução significativa na média do tempo de internamento após a implementação do protocolo, evidenciando uma relação positiva entre a implantação de medidas preventivas para o desenvolvimento de LPP e a redução do tempo de internamento ${ }^{1,14,20}$. A partir da mudança de comportamento por meio da adoção de atitudes voltadas à prevenção pela equipe multiprofissional, resultados positivos na qualidade da assistência prestada são obtidos ${ }^{3,6}$.

No que se refere à incidência de LPP na UTI, houve uma redução significativa no surgimento de LPP após a implantação do protocolo, nos últimos trimestres, dos anos avaliados. Desta forma, é possível inferir que o uso de protocolos como ferramenta preventiva, somado à mudança de comportamento assistencial e capacitação profissional, representa fatores determinantes na redução da incidência de LPP e eventos adversos, ${ }^{9,421-22}$.

A baixa incidência de LPP em hospitais é tida como indicador de qualidade do serviço. Mudança de indicadores certifica a qualidade da assistência e de profissionais, umavez que nãoépossível ter assistência de qualidade sem profissionais capacitados, competentes e qualificados $^{10}$. A legislação brasileira 
determina a atuação de um enfermeiro para cada dez leitos e um técnico em enfermagem para cada dois leitos de UTI ${ }^{11,22-23}$. Porém, este dimensionamento nem sempre é suficiente para execução de uma assistência de qualidade, requerendo um quantitativo maior de profissionais diminuindo assim o desgaste, a sobrecarga e o estresse dos mesmos.

Além das questões assistenciais, existem os fatores intrínsecos aos pacientes tais como suas condições clínica e nutricional e os fatores extrínsecos, como, por exemplo: presença de umidade; cisalhamento e fricção na pele do paciente; além das condições estruturais e materiais de assistência, que devem ser levadas em consideração no desenvolvimento de $\mathrm{LPP}^{4}$.

A hipótese diagnóstica apresentou diferença a partir da implantação do protocolo na UTI, onde a prevalência passou da categoria causas externas para categoria cirúrgica. Esta diferença pode ter relação com o perfil dos pacientes admitidos na UTI. O aumento do número de pacientes com hipótese diagnóstica cirúrgica pode ter relação com a impossibilidade de mobilização do paciente devido ao procedimento cirúrgico, independente de aplicação de medidas preventivas.

A aplicação preventiva de coxins nos últimos trimestres dos anos avaliados revelou uma associação significativa na redução do uso destes dispositivos na UTI, evidenciando a eficácia da aplicação do protocolo, visto que, no início da aplicação do mesmo, a equipe fazia uso de coxins nos locais que posteriormente foram substituídos pelos aliviadores de pressão na região calcânea, e de placas de hidrocoloide regular na região sacra.

Porém, estes resultados não eximem a necessidade do uso de superfícies de suporte que auxiliem na prevenção e tratamento de LPP, apenas ratifica a importância de medidas preventivas em conjunto, visando a uma assistência mais completa possível, assim como recomendado na literatura, tais como uso de coxins, mudança de decúbito, colchões piramidais e/ou pneumáticos, hidratação e higiene da pele ${ }^{5,24-26}$.

Embora os resultados tenham revelado uma redução global no surgimento de LPP em todas as regiões corporais durante o internamento, as regiões calcânea e sacra apresentaram diferença significativa na incidência após a implantação do protocolo. Medidas que, de uma forma geral, promovam a redução da pressão nas proeminências ósseas, independente da região corporal, são largamente reconhecidas e recomendadas ${ }^{21,24,26}$.

Esta pesquisa foi relevante, visto que a implantação do protocolo na unidade possibilitou um direcionamento e consequente mudança nas ações da equipe multiprofissional, resultando em benefícios não só com relação à prevenção de LPP, mas também à qualidade da assistência. A limitação encontrada para a realização desta pesquisa foi relacionada ao preenchimento inadequado ou incompleto de algumas informações tanto nos livros de registro como nos protocolos, especialmente no último trimestre de 2015.

\section{CONCLUSÕES}

Foi comprovada a efetividade da implantação do protocolo para prevenção de LPP na UTI analisada, a partir dos resultados que evidenciaram uma redução significativa no surgimento de LPP durante o internamento. As variáveis: tempo de internamento; hipótese diagnóstica; aplicação preventiva de coxins; e o surgimento de LPP nas regiões calcânea e sacra, apresentaram diferenças significativas, após a implementação do protocolo.

\section{REFERÊNCIAS}

Alencar GSA, Silva NM, Assis EV, Sousa MNA, Pereira JLF, Oliveira WB, et al. Lesão por pressão na unidade de terapia intensiva: incidência e fatores de riscos. Rev Nursing [Internet]. 2018 [cited 2018 Jun 06]; 21(239):2124-8. Available from: http:// www.revistanursing.com.br/revistas/239-Abril2018/lesao_por_ 
pressao_na_unidade_de_terapia_intensiva.pdf.

2. Sousa LRM. Prevention and treatment of pressure ulcers: brazilian literature Analysis. Rev Enferm UFPI [Internet]. 2015 [cited 2018 Feb 12]; 4(3):79-85. Available from: http://www.ojs. ufpi.br/index.php/reufpi/article/view/1983/pdf

3. National Pressure Ulcer Advisory Panel EPUAP e PPPIA. Prevenção e Tratamento de Úlceras por Pressão: Guia de Consulta Rápida [Internet]. 2014 [cited 2018 Mar 03] Available from: http://sociedadeferidas.pt/documentos/Prevencao_e_ Tratamento_de_Ulceras_Por_Pressao-Guia_de_Referencia_ Rapido.pdf.

4. Moraes JT, Borges EL, Lisboa CR, Cordeiro DCO, Rosa EG, Rocha NA. Conceito e classificação de lesão por pressão: atualização do National Pressure Ulcer Advisory Panel. Enferm Cent O Min. 2016 [cited 2018 Feb 12]; 6(2):2292-306. Available from: http://dx.doi.org/10.19175/recom.v6i2.1423.

5. Meyers T. Prevention of heel pressure injuries and plantar flexion contractures with use of a heel protector in highrisk neurotrauma, medical, and surgical intensive care units. JWOCN. 2017 [cited 2018 Apr 20]; 44(5):429-33. Available from: http://dx.doi.org/10.1097/WON.00000000000000355.

6. Agência Nacional de Vigilância sanitária (ANVISA). Práticas seguras para prevenção de Lesão por Pressão em serviços de saúde. Nota técnica GVIMS/GGTES n. 03/2017. [Internet] 2017. Available from: https://www20.anvisa.gov.br/ segurancadopaciente/index.php/alertas/item/nota-tecnicagvims-ggtes-03-2017.

7. Ministério da Saúde (BR). PORTARIA No 529, de 1o de abril de 2013. Institui o Programa Nacional de Segurança do Paciente (PNSP). Resolução n. 529/2013. Brasília: Ministério da Saúde, 2013.

8. Vasconcelos JMB, Caliri MHL. Nursing actions before and after a protocol for preventing pressure injury in intensive care. Esc Anna Nery. 2017 [cited 2018 Apr 20]; 21(1):1-9. Available from: http://dx.doi.org/10.5935/14148145.20170001.

9. Soares RSA, Lima SBS, Camponogara S, Eberhardt TD, Fonseca GGP da, Kessler M. Significado do protocolo de úlcera por pressão:qualificando a gerência do cuidado do enfermeiro. Enferm. Foco. 2017 [cited 2018 Jun 06];8(3):19-24. http://dx.doi. org/10.21675/2357-707X.2017.v8.n3.859.

10. Ministério da Saúde (BR). Aprova os protocolos básicos de segurança do paciente. Portaria n. 2.095/2013. Brasília: Ministério da Saúde, 2013.

11. Ministério da Saúde (BR). Dispõe sobre os requisitos mínimos para funcionamento de unidades de terapia intensiva e dá outras providências. Resolução n. 7/2010. Brasília: Ministério da Saúde, 2010

12. Alderden J, Cummins MR, Pepper GA, Whitney JD, Zhang $\mathrm{Y}$, Butcher R, et al. Midrange Braden subscale scores are associated with increased risk for pressure injury development among critical care patients. JWOCN. 2017 [cited 2018 Mar 03];44(5):420-28. Available from: http://dx.doi.org/10.1097/ WON.0000000000000349.

13. Mehta C, George J V, Mehta Y, Wangmo N. Pressure ulcer and patient characteristics e a point prevalence study in a tertiary hospital of India based on the European Pressure Ulcer Advisory Panel minimum data set. J Tissue Viability. 2015 [cited 2018 Mar 03]; 24(3):123-30. Available from: http:// dx.doi.org/10.1016/j.jtv.2015.04.001.

14. Campanili TCGF, Santos VLCG, Strazzieri-Pulido KC, Thomaz PBM, Nogueira PC. Incidência de úlcera por pressão em pacientes de unidade de terapia intensiva cardiopneumológica*. Rev Esc Enferm USP. 2015 [cited 2018 Feb 12]; 49(Esp):7-14. Available from: http://dx.doi. org/10.1590/S0080623420150000700002.

15. Kock KS, Guadagnin F, Machado MO, Maurici R. Comparação do APACHE II e PCR como preditores de mortalidade em pacientes hospitalizados em UTI. Rev da AMRIGS [Internet]. 2014 [cited 2018 Mar 08]; 58(3):182-6. Available from: http://www.amrigs.org.br/revista/58-03/002.pdf.

16. Andrade SSCA, Jorge MHPM. Hospitalization due to road traffic injuries in Brazil, 2013: hospital stay and costs*. Epidemiol Serv Saúde. 2017 [cited 2018 Mar 08]; 26(1). Available from: http://dx.doi.org/10.5123/S167949742017000100004.

17. Gomes ATL, Silva MF, Dantas, BAS, Miranda JMA, Melo GSM, Dantas RAN. Perfil epidemiológico das emergências traumáticas assistidas por um serviço pré-hospitalar móvel de urgência. Enfermería Global. 2017 [cited 2018 Jun 06]; 45:395-405. Available from: http://dx.doi.org/10.6018/ eglobal.16.1.231801.

18. Alves AGP, Borges JWP, Brito MA. Avaliação do risco para úlcera por pressão em unidades de terapia intensiva: uma revisão integrativa. J res fundam care. 2014 [cited 2018 Jun 06];6(2):793-804. Available from: http://dx.doi.org/10.9789/21755361.2014v6n2p793.

19. Barbosa TP, Beccaria LM, Poletti NAA. Avaliação do risco de úlcera por pressão em UTI e assistência preventiva de enfermagem. Rev Enferm UERJ [Internet]. 2014 [cited 2018 Apr 06]; 22(3):353-8. Available from: http://www.facenf.uerj.br/ v22n3/v22n3a10.pdf.

20. Dutra RAA, Salomé GM, Leal LM, Alves MG, Moura JP, Silva AT, et al. Cost comparison of pressure ulcer preventive dressings: hydrocolloid dressing versus transparent polyurethane film. J Wound Care. 2016 [cited 2018 Mar 03]; 25(11):635-40. Available from: http://dx.doi.org/10.12968/ jowc.2016.25.11.635.

21. Pickham D, Ballew B, Ebong K, Shinn J, Lough ME, Mayer B. Evaluating optimal patient-turning procedures for reducing hospital-acquired pressure ulcers (LS-HAPU): study protocol for a randomized controlled trial. BioMed Cent. 2016 [cited 2018 Mar 03]; 17(190):1-8. Available from: http://dx.doi. org/10.1186/s13063-016-1313-5.

22. Ministério da Saúde (BR). Altera a Resolução RDC $n^{\circ}$ 07/2010, que dispõe sobre os requisitos mínimos para funcionamento de unidades de terapia intensiva e dá outras providências. Resolução RDC n. 26/2012. Brasília: Ministério da Saúde, 2012.

23. Ministério da Saúde (BR). Atualiza e estabelece parâmetros para o dimensionamento do quadro de profissionais de 
enfermagem nos serviços/locais em que são realizadas atividades de enfermagem. Resolução COFEN n. 542/2017. Brasília: Ministério da Saúde, 2017.

24. Serraes B, Beeckman D. Static air support surfaces to prevent pressure injuries: a multicenter cohort study in Belgian nursing homes. Wound, Ostomy Cont Nurses Soc. 2016 [cited 2018 Mar 03]; 43(4):375-8. Available from: http:// dx.doi.org/10.1097/WON.0000000000000244.

25. Avsar P, Karadag A. Efficacy and cost-effectiveness analysis of evidence-based nursing interventions to maintain tissue integrity to prevent pressure ulcers and incontinenceassociated dermtitis. Worldviews Evidence-Based Nurs. 2018 [cited 2018 Jun 08]; 15(1):54-61. Available from: http:// dx.doi.org/10.1111/wvn.12264.

26. Edger M. Effect of a patient-repositioning device in an intensive care unit on hospital-acquired pressure injury occurences and cost. Wound, Ostomy Cont Nurses Soc. 2017 [cited 2018 Jun 08]; 44(3):236-40. Available from: http:// dx.doi.org/10.1097/WON.0000000000000328. 